\title{
Broad-Band Radial Slot Antenna Fed by Coplanar Waveguide for Dual-Frequency Operation
}

\author{
Shih-Yuan Chen and Powen Hsu, Senior Member, IEEE
}

\begin{abstract}
A novel design of a broad-band radial slot antenna fed by a coplanar waveguide for dual-frequency operation is presented. Various frequency ratios, within the range of about 1.3 to 2.1, of the two operating frequencies can be obtained by varying the included angle between the radial slots and/or by varying the length of the central slot pair. The bandwidth of the lower operating band lies in the range of $2.4 \%$ to $9.7 \%$, while that of the upper band is much wider and ranges from $17.4 \%$ to $23.2 \%$. Radiation patterns at the two operating frequencies are broadside and bidirectional. Details of the antenna design and the experimental results are presented and discussed.
\end{abstract}

Index Terms-Coplanar waveguides, multifrequency antennas, slot antennas.

\section{INTRODUCTION}

T HERE have been growing research activities on dual-band and multiband conformal antennas for integrations of heterogeneous communication systems such as the global system for mobile communication (GSM), the general packet radio service (GPRS), the global position system (GPS), the wireless local area networks (WLAN), and the universal mobile telecommunications system (UMTS). Among them, the microstrip patch antenna and the slot antenna are two of the most prevailing antenna structures. Several different designs of dual-frequency microstrip patch antenna have been proposed previously, such as the stacked microstrip antenna [1], the reactively loaded microstrip antenna, the dual-mode (operated at $\mathrm{TM}_{10}, \mathrm{TM}_{01}$ or other higher order modes) microstrip patch antenna [2]-[5], and the planar inverted-F antenna [6]-[8]. These designs, however, show high-Q characteristics in both operating bands with their bandwidths in the range of about $2 \%$ due to the microstrip configuration. Instead, slot antennas have broader impedance bandwidths than microstrip patch antennas. Moreover, slot antennas radiate bidirectionally while microstrip patch antennas radiate in one direction. In some wireless and spatial power combing applications, bidirectional antennas are more useful [9]. In spite of these advantages, there are fewer slot-based dual-band antennas proposed so far. Slot ring and slot loop antennas were commonly the fundamental structures used in [10]-[12], and the slot spiral antenna was proposed in [13].

Manuscript received March 7, 2004; revised March 21, 2005. This work was supported in part by the Ministry of Education, Taiwan, R.O.C., under Contract 89-E-FA06-2-4 and in part by the National Science Council, Taiwan, R.O.C., under Contract NSC 93-2752-E-002-004-PAE.

The authors are with the Department of Electrical Engineering and Graduate Institute of Communication Engineering, National Taiwan University, Taipei 106, Taiwan, R.O.C. (e-mail: phsu@cc.ee.ntu.edu.tw).

Digital Object Identifier 10.1109/TAP.2005.858574

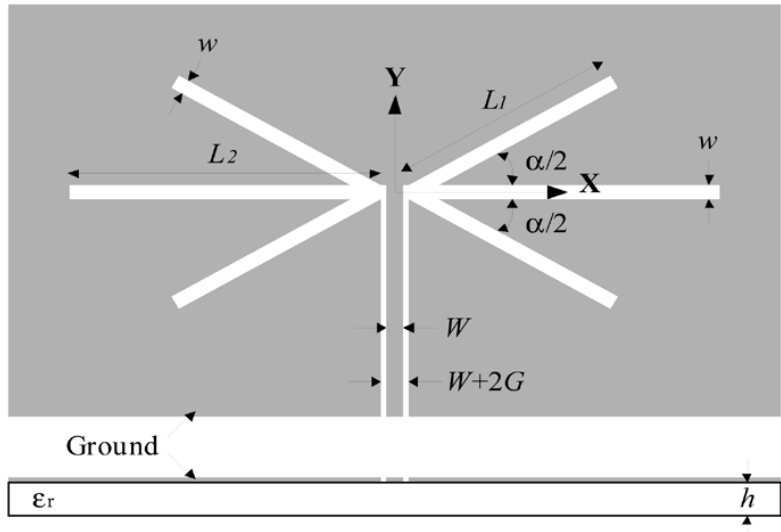

Fig. 1. Geometry of the CPW-fed radial slot antenna.

In this paper, a novel coplanar waveguide (CPW) fed radial slot antenna is proposed for dual-frequency operation. The frequency ratio of the two operating frequencies can be controlled by varying the included angle between the radial slots. Finetuning of the frequency ratio can be further obtained by varying the length of the central slot. The frequency ratio of the proposed design is tunable in the range of 2.10 to 1.30 , while that of the slot antenna discussed in [12] ranges from 1.47 to 1.10 . The two operating bands of the proposed design have similar radiation properties, including their polarizations and patterns, and the measured impedance bandwidths for the lower and upper operating bands are wide and range from 2.4 to $9.7 \%$ and 17.4 to $23.2 \%$, respectively. In the upper operating band, not only the bandwidth is wider, but the cross-polarization radiation levels in both principle planes are far lower than those of the slot ring and the slot loop antennas proposed in [10] and [11], respectively. Also, the uni-planar and simple structure of the proposed design make it ease of mass production and more cost-effective than the cavity-backed slot-spiral antenna presented in [13]. Details of the design are presented. Experimental and simulated results of the obtained dual-frequency characteristics are also presented and discussed.

\section{ANTENNA CONFIGURATION AND OPERATION}

The configuration of the CPW-fed radial slot antenna is shown in Fig. 1. The central pair of the slots with dimension of $L_{2} \times w$ is normal to the CPW feedline. All of the other four oblique slots having the same dimension of $L_{1} \times w$ are oriented to have an angle $\alpha / 2$ measured from the central slot. The widths of the strip and slot of the feeding CPW are $W$ and $G$, respectively. As shown in Fig. 1, the geometry symmetric to 


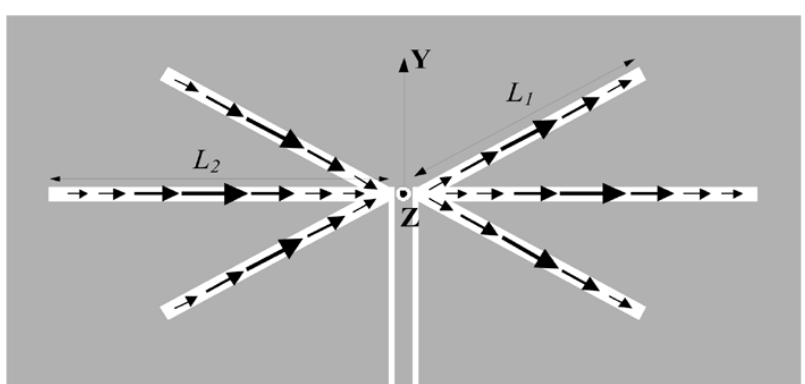

(a)

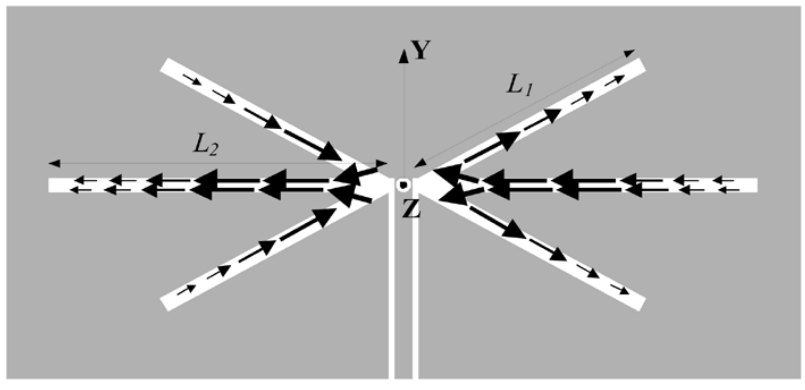

(b)

Fig. 2. Equivalent magnetic current distributions on radial slot antenna with $L_{1}<L_{2}$ and operating at (a) $f_{H}$ and (b) $f_{L}$.

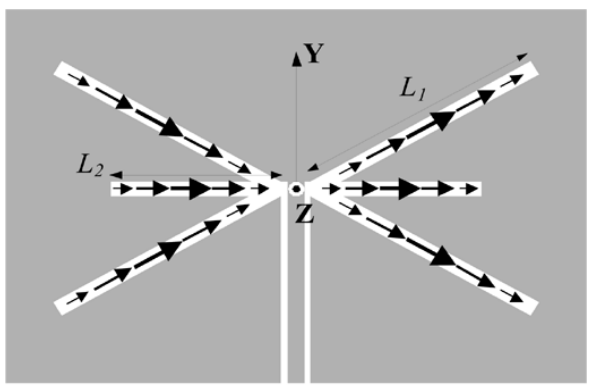

(a)

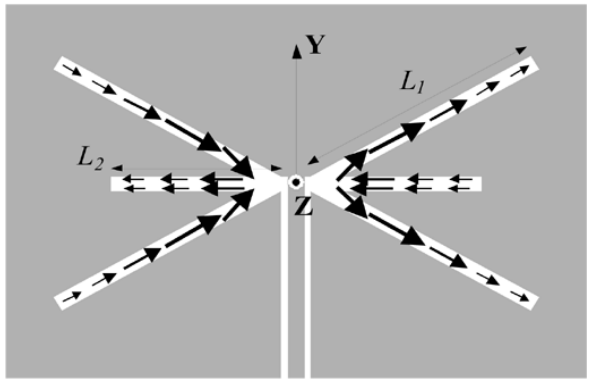

(b)

Fig. 3. Equivalent magnetic current distributions on radial slot antenna with $L_{1}>L_{2}$ and operating at (a) $f_{H}$ and (b) $f_{L}$.

the $x$ - and $y$-axes results in less complexity during the design process, and the uniplanar structure makes it ease of fabrication.

The principles of the dual-frequency operation of this antenna can be observed via Figs. 2 and 3. These figures are obtained from the electromagnetic simulator Ansoft Ensemble. ${ }^{1}$ The instantaneous equivalent surface magnetic current distributions of the radial slot antennas at resonance with $L_{1}<L_{2}$ and $L_{1}>L_{2}$

${ }^{1}$ Ansoft Ensemble is a registered trademark of Ansoft Corporation, Pittsburgh, PA 15219-1119 USA. are shown in Figs. 2 and 3, respectively. As shown in Fig. 2 $\left(L_{1}<L_{2}\right)$, two resonant frequencies $f_{H}$ and $f_{L}$ exist, where $f_{H}$ and $f_{L}$ stand for the higher and lower resonant frequencies, respectively. When operating at $f_{H}$ [Fig. 2(a)], more equivalent magnetic currents are flowing on the oblique slots and the resonant frequency $f_{H}$ is mainly determined by $L_{1}$. $L_{1}$ is slightly smaller than half the guided-wavelength $\lambda_{g} / 2$ in the structure and $L_{2}$ is larger than $\lambda_{g} / 2$. All the magnetic currents on the radial slots can be decomposed into $x$ - and $y$-components. In Fig. 2(a), the in-phase $x$-components produce a broadside bidirectional radiation pattern at resonance, while the $y$-components cancel out each other along the $x$ and $y$-axes which result in a low cross polarization radiation level in both principle planes $\left(\phi=0^{\circ}\right.$ and $\phi=90^{\circ}$ ). The cross-polarization level will reach its maximum in the plane of $\phi=45^{\circ}$ but remain acceptable. When operating at $f_{L}$ [Fig. 2(b)], $L_{1}$ plus $L_{2}$ equals to $\lambda_{g} / 2$, and more magnetic current will distribute on the central slot pair for $L_{2}$ longer than $L_{1}$. Although the $x$-components of the magnetic current distributed on the oblique slots are out-of-phase with those of the central slot pair, the amount of these components can only slightly lower the antenna gain but will not affect the radiation pattern enormously. Thus, the pattern distortion of the co-polarization components in other oblique planes different from $\mathrm{E}$ and $\mathrm{H}$ planes is negligible.

In Fig. 3(a), where $L_{1}>L_{2}$ and the antenna is operating at $f_{H}, L_{1}$ is slightly longer than $\lambda_{g} / 2$ and $L_{2}$ is shorter than $\lambda_{g} / 2$. The resonant frequency $f_{H}$ is mainly determined by $L_{1}$. The radiation pattern resembles that of the case in Fig. 2(a). On the other hand, Fig. 3(b) shows that, when operating at $f_{L}$, where $L_{1}$ plus $L_{2}$ equals to $\lambda_{g} / 2$, there is more magnetic current distributed on the oblique slots than on the central slot pair, and the $x$-component of the magnetic current on the oblique slots will cancel out with that on the central slot pair and results in a poor radiation pattern. Therefore, in the present proposed design, $L_{2}$ is recommended to be longer than $L_{1}$ to ensure satisfactory radiation patterns at both resonant frequencies. Furthermore, the case with $L_{1} \approx L_{2}$ is a broad-band design with $L_{1}$ and $L_{2}$ both approximately equal to half the guided-wavelength at resonance. The $x$-components of the magnetic current on all slots are in phase to have a broadside and bidirectional radiation pattern, while the $y$-components cancel out each other to ensure a low cross polarization level. Note that the wide bandwidth nature of this case with $L_{1} \approx L_{2}$ can be further increased by letting $L_{1}<L_{2}$ or $L_{1}>L_{2}$. However, as described above for Figs. 2 and 3 , there would be an additional lower frequency band occurs when the difference between $L_{1}$ and $L_{2}$ becomes significant. $L_{1}$ is approximately equal to half the guided-wavelength at the inherent resonant frequency of the design with $L_{1} \approx L_{2}$, and this is also the case for the upper resonant frequency $f_{H}$ in Figs. 2(a) and 3(a). In addition, the magnetic current distributions at resonance resemble those illustrated in Figs. 2(a) and 3(a). Thus, the upper operating band near $f_{H}$ of the dual-frequency operation in Figs. 2(a) and 3(a) corresponds to the inherent operating band of the broad-band design with $L_{1} \approx L_{2}$, hence the bandwidth of the upper operating band near $f_{H}$ is much wider than that of the lower band near $f_{L}$. It is worth mentioning that the enhanced bandwidth of the upper band of the proposed design 


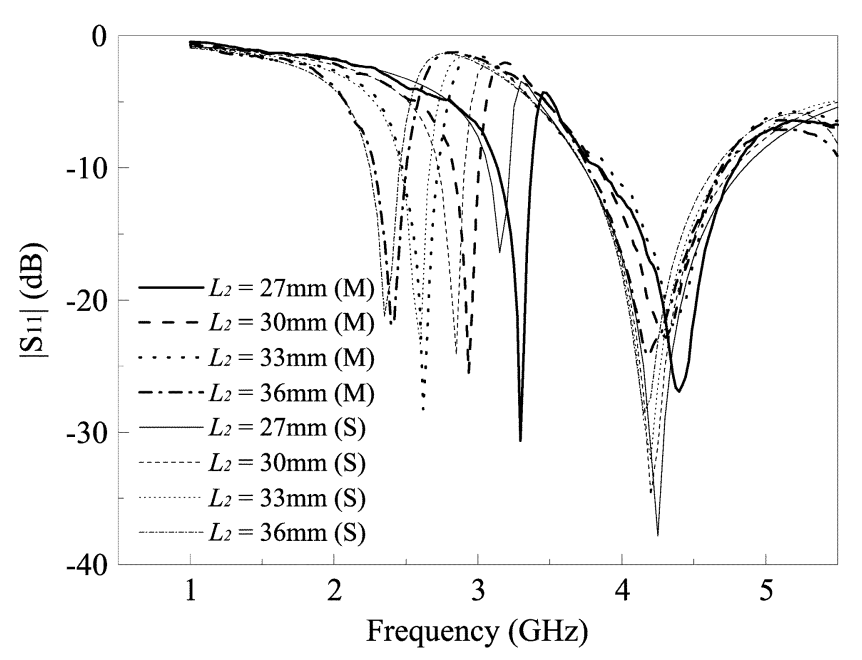

(a)

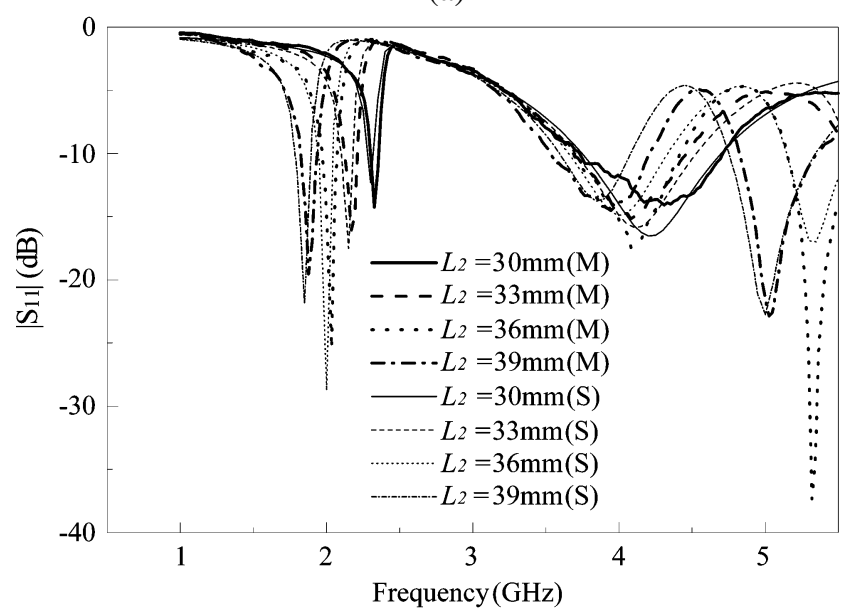

(b)

Fig. 4. Measured and simulated input return losses of the radial slot antenna with (a) $\alpha=30^{\circ}$ and (b) $\alpha=60^{\circ}$ for different $L_{2}$. ( $M$ : measured, $S$ : simulated).

as shown in Fig. $2\left(L_{1}<L_{2}\right)$ is satisfactory for most wireless applications.

\section{ANTENNA DESIGN AND EXPERIMENTAL RESULTS}

Several typical designs of the proposed antenna with various lengths of the central slot pair $L_{2}$ and included angles $\alpha$ were constructed and investigated. All of the test pieces were fabricated on the dielectric substrate FR-4 with dielectric constant $\varepsilon_{r}=4.3$, substrate thickness $h=1.6 \mathrm{~mm}$, and loss tangent $\tan \delta=0.02$. The widths of the strip and slot, $W$ and $G$, of the $50 \Omega \mathrm{CPW}$ feedline are fixed to be 3.4 and $0.3 \mathrm{~mm}$, respectively, and the length of the oblique slots $L_{1}$ is fixed to be $23.1 \mathrm{~mm}$, which is approximately half of the guided-wavelength in the structure at $4.5 \mathrm{GHz}$. The width of the oblique slots and the central slot pair $w$ is one of the key parameters to tune for the input match of the antenna because it has a negligible effect on the resonant frequencies and the frequency ratio. In the test pieces, $w$ is chosen to be 2.4 or $2.6 \mathrm{~mm}$ for a compromised matching at resonant frequencies. A series of designs with various $L_{2}(27$, $30,33,36$, and $39 \mathrm{~mm})$ and $\alpha\left(30^{\circ}\right.$ and $\left.60^{\circ}\right)$ were implemented and tested.
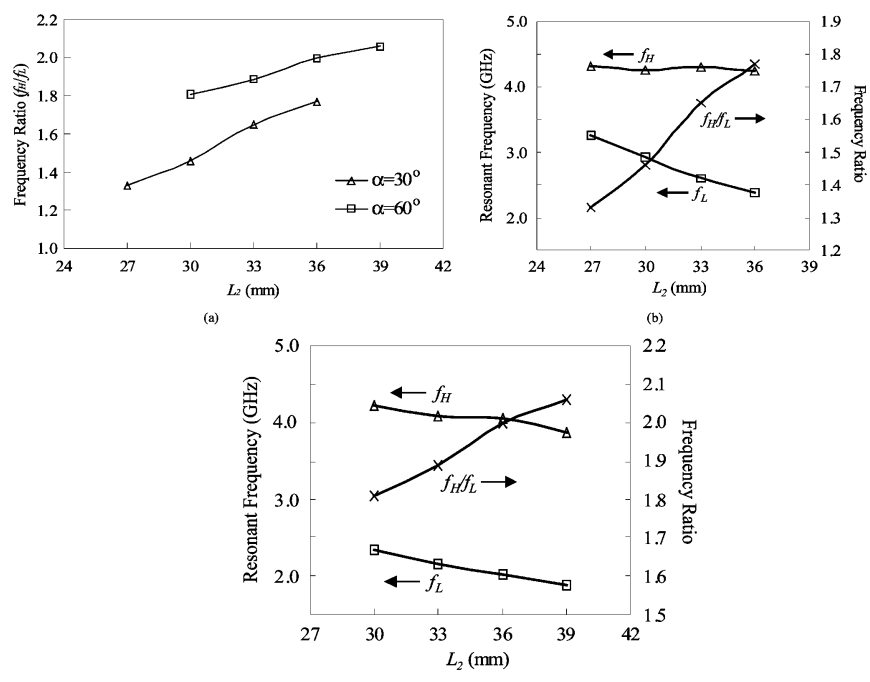

(c)

Fig. 5. Measured frequency ratio $\left(f_{H} / f_{L}\right)$ and resonant frequencies $\left(f_{H}\right.$ and $f_{L}$ ) against $L_{2}$. (a) $f_{H} / f_{L}$ versus $L_{2}$, (b) $f_{H}, f_{L}$, and $f_{H} / f_{L}$ versus $L_{2}$ for $\alpha=30^{\circ}$, and (c) $f_{H}, f_{L}$, and $f_{H} / f_{L}$ versus $L_{2}$ for $\alpha=60^{\circ}$.

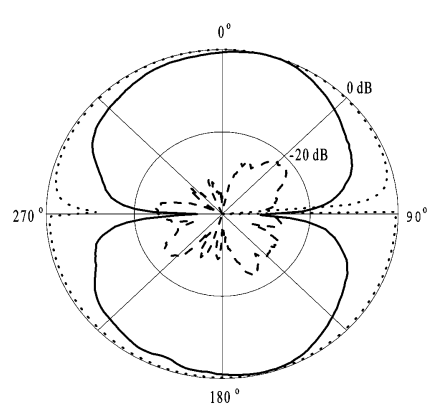

(a)

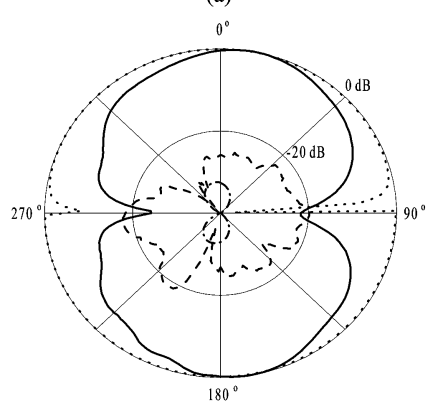

(c)

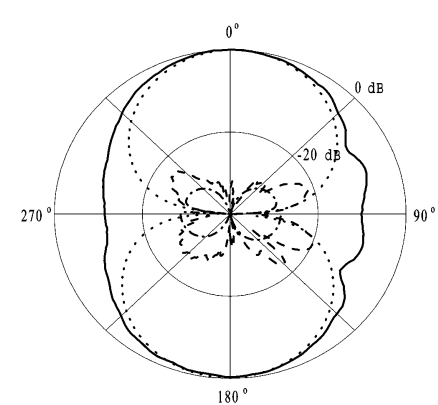

(b)

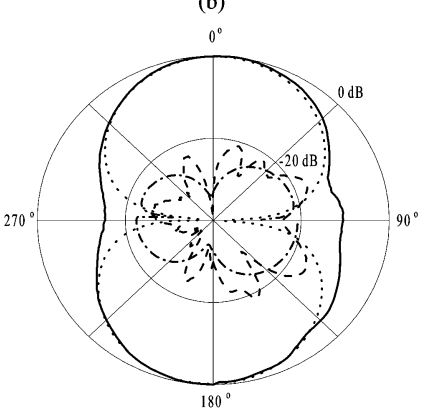

(d)
Fig. 6. Measured and simulated radiation patterns with $L_{2}=27.0 \mathrm{~mm}$ and $\alpha=30^{\circ}$. (a) E-plane pattern at $f_{H}$, (b) H-plane pattern at $f_{H}$, (c) E-plane pattern at $f_{L}$, and (d) H-plane pattern at $f_{L}$. - Measured co-pol, - - - Measured cross-pol, ......... Simulated co-pol, . . . - Simulated cross-pol.

Measured return losses of the test pieces and the ones simulated by Ansoft Ensemble 6.0 are shown in Fig. 4. They are in good agreement. The summary of the experimental frequency response results of Fig. 4 is depicted in Fig. 5. The measured impedance bandwidth, defined as a return loss level of $10 \mathrm{~dB}$, of the upper band falls in the range from $17.4 \%$ to $23.2 \%$, while that of the lower band ranges from $2.4 \%$ to $9.7 \%$. The bandwidth of the lower band decreases as the included angle $\alpha$ increases. It is worthy mentioning that, in the course of simulation, we found that the effective length that the equivalent magnetic current actually flows at $f_{L}$ along the slots elongates for 


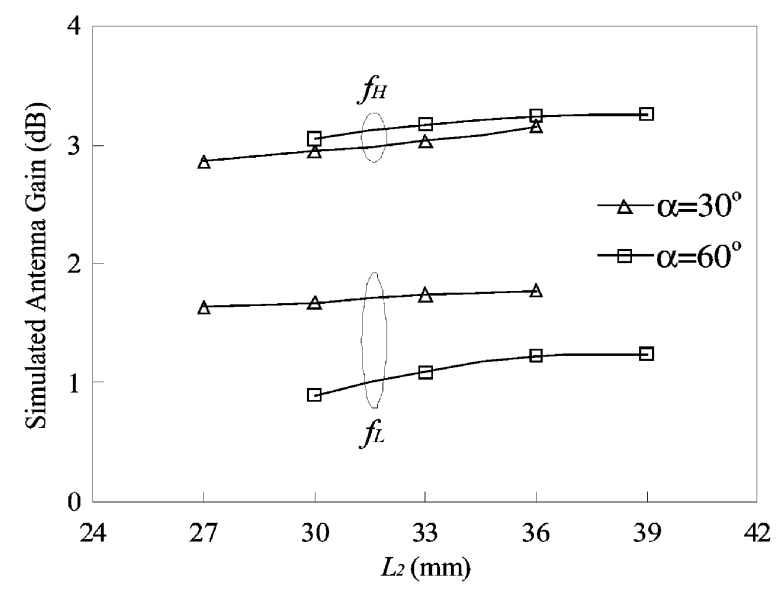

Fig. 7. Antenna gains simulated at $f_{H}$ and $f_{L}$ against $L_{2}$ for various $\alpha$.

larger included angle $\alpha$ even though the physical slot lengths remain unchanged. Thus, the lower resonant frequency $f_{L}$ decreases and the frequency ratio increases monotonically as the included angle $\alpha$ increases. Consequently, there is a tradeoff between the frequency ratio and the bandwidth of the lower band, which sets an upper limit of the frequency ratio for the design. The obtained frequency ratio for the present design varies in the range of about 1.3 to 2.1 and increases monotonically with the increasing $L_{2}$. On the other hand, the resonant frequencies $f_{H}$ and $f_{L}$ decrease as $L_{2}$ increases, and $f_{L}$ is more sensitive to the variation of $L_{2}$ than $f_{H}$.

The difference between radiation patterns of the antennas with various $L_{2}$ and $\alpha$ is insignificant. Only one case of the radiation pattern will be shown. The measured and simulated radiation patterns for the radial slot antenna with $L_{2}=27.0 \mathrm{~mm}$, $\alpha=30^{\circ}$, and operating at $f_{H}$ and $f_{L}$ are shown in Fig. 6 . Note that the cross polarization level at $f_{H}$ is lower than that at $f_{L}$, which agrees well with the descriptions in Section II. The radiation patterns at $f_{H}$ and $f_{L}$ are of the same polarization. The antenna gains measured at $f_{H}$ and $f_{L}$ are 4.4 and $3.1 \mathrm{dBi}$, respectively, while those simulated at $f_{H}$ and $f_{L}$ are 2.86 and $1.64 \mathrm{~dB}$, respectively. The antenna gains simulated at both resonant frequencies against $L_{2}$ for various $\alpha$ are shown in Fig. 7. The antenna gain at $f_{L}$ is lower than that at $f_{H}$ is due to the field cancellation between the central slot pair and the oblique slots. and demonstrated the principle of the dual-frequency operation. The behavior of the antenna has been studied intensively and investigated experimentally. The frequency ratio of the two resonant frequencies is tunable from 1.3 to 2.1 by varying $\alpha$ and $L_{2}$. The bandwidth of the lower band ranges from 2.4 to $9.7 \%$, and a much wider bandwidth of the upper band ranges from 17.4 to $23.2 \%$. This antenna may find applications in many wireless communication systems.

\section{REFERENCES}

[1] S. A. Long and M. D. Walton, "A dual-frequency stacked circular-disc antenna," IEEE Trans. Antennas Propag., vol. AP-27, no. 2, pp. 270-273, Mar. 1979.

[2] B. F. Wang and Y. T. Lo, "Microstrip antennas for dual-frequency operation," IEEE Trans. Antennas Propag., vol. AP-32, no. 9, pp. 938-943, Sep. 1984.

[3] W. F. Richards, S. E. Davidson, and S. A. Long, "Dual-band reactively loaded microstrip antenna," IEEE Trans. Antennas Propag., vol. AP-33, no. 5, pp. 556-561, May 1985.

[4] S. C. Pan and K. L. Wong, "Dual-frequency triangular microstrip antenna with a shorting pin," IEEE Trans. Antennas Propag., vol. 45, no. 12, pp. 1889-1891, Dec. 1997.

[5] S. C. Gao, L. W. Li, T. S. Yeo, and M. S. Leong, "Small dual-frequency microstrip antennas," IEEE Trans. Veh. Technol., vol. 51, no. 1, pp. 28-36, Jan. 2002.

[6] T. Kashiwa, N. Yoshida, and I. Fukai, "Analysis of radiation characteristics of planar inverted-F type antenna on conductive body of hand-held transceiver by spatial network method," Electron. Lett., vol. 25, no. 16, pp. 1044-1045, Aug. 1989.

[7] C. R. Rowell and R. D. Murch, "A capacitively loaded PIFA for compact mobile telephone handsets," IEEE Trans. Antennas Propag., vol. 45, no. 5, pp. 837-842, May 1997.

[8] Z. D. Liu, P. S. Hall, and D. Wake, "Dual-frequency planar inverted-F antenna," IEEE Trans. Antennas Propag., vol. 45, no. 10, pp. 1451-1458, Oct. 1997.

[9] J. A. Navarro and K. Chang, Integrated Active Antennas and Spatial Power Combining. New York: Wiley, 1996.

[10] H. Tehrani and K. Chang, "Multifrequency operation of microstrip-fed slot-ring antennas on thin low-dielectric permittivity substrates," IEEE Trans. Antennas Propag., vol. 50, no. 9, pp. 1299-1308, Sep. 2002.

[11] D. Llorens, P. Otero, and C. Camacho-Penalosa, "Dual-band, single CPW port, planar-slot antenna," IEEE Trans. Antennas Propag., vol. 51, no. 1, pp. 137-139, Jan. 2003.

[12] W. S. Chen and K. L. Wong, "A dual-frequency coplanar waveguide-fed slot antenna," Microwave Opt. Technol. Lett., vol. 25, no. 3, pp. 226-228, May 2000.

[13] D. S. Filipovic and J. L. Volakis, "Novel slot spiral antenna designs for dual-band/multiband operation," IEEE Trans. Antennas Propag., vol. 51, no. 3, pp. 430-440, Mar. 2003.

\section{CONCLUSION}

A novel broad-band, dual-frequency radial slot antenna fed by a CPW has been presented in this paper. This antenna simultaneously possesses a wider bandwidth of the upper band, satisfactory radiation patterns, high radiation efficiency, and compact size. In addition, the uniplanar and simple structure makes it ease of fabrication and design. Distributions of the equivalent magnetic current on the radial slot antenna have been shown

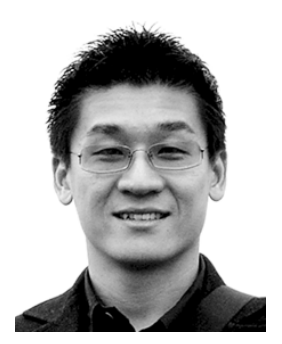

Shih-Yuan Chen was born in Chang-Hua, Taiwan, R.O.C., in 1978. He received the B.S. degree in electrical engineering and the M.S. and Ph.D. degrees in communication engineering from the National Taiwan University, Taipei, Taiwan, R.O.C., in 2000, 2002, and 2005, respectively.

Since August 2005, he has been a Postdoctorate Research Fellow with the Graduate Institute of Communication Engineering, National Taiwan University. His current research interests include the design and analysis of slot antennas, dielectric lens antennas, microstrip antennas, and RFID tag antennas. 
3452

IEEE TRANSACTIONS ON ANTENNAS AND PROPAGATION, VOL. 53, NO. 11, NOVEMBER 2005

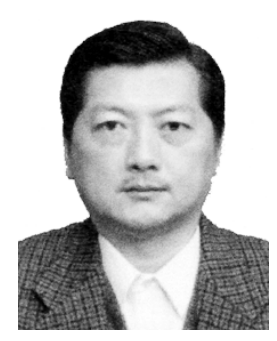

Power Hsu (M'86-SM'98) was born in Taipei, Taiwan, R.O.C., in 1950. He received the B.S. degree in physics from the National Tsing-Hua University, Hsinchu, Taiwan, R.O.C., in 1972, the M.S. degree in physics from the University of Maryland, College Park, in 1976, and the M.S. and Ph.D. degrees in electrical engineering from the University of Southern California, Los Angeles, in 1978 and 1982, respectively.

From 1982 to 1984, he was with ITT Gilfillan, Van

Nuys, CA, where he was engaged in research and development pertaining to radar antenna systems. In 1984, he joined the faculty of the National Taiwan University, Taipei, Taiwan, R.O.C., where he is currently a Professor with the Electrical Engineering Department. From 1992 to 1995, he was the Department Chairperson at the same university. In August 1997, he established the ninth college, College of Electrical Engineering and Computer Science, in the National Taiwan University, and served as the first Dean of the College until 2003. His current research interests include the design and analysis of slot antennas, microstrip antennas, RFID tag antennas, and microwave and millimeter-wave integrated circuits.

Authorized licensed use limited to: IEEE Xplore. Downloaded on February 18, 2009 at 03:07 from IEEE Xplore. Restrictions apply. 